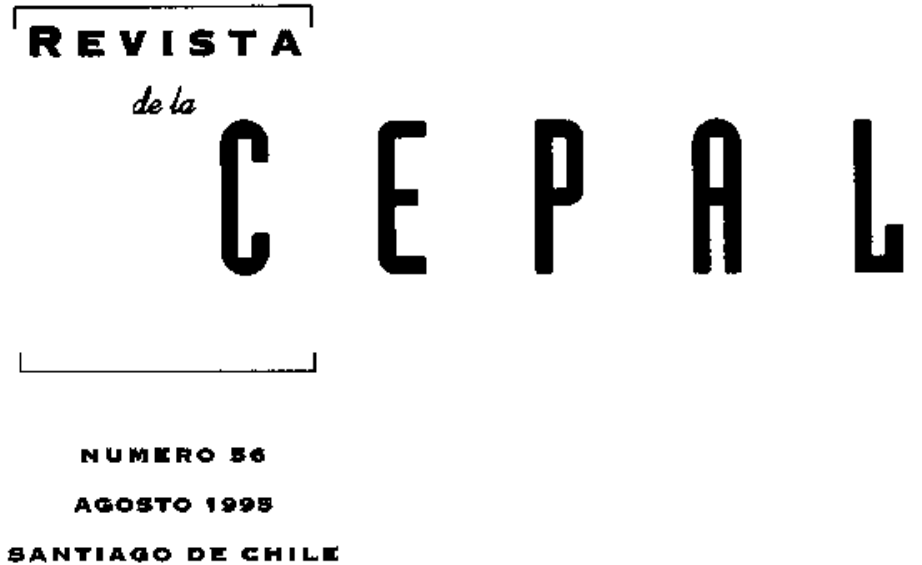

ANIBAL PINTO

Director

EUGENIO LAHERA

Secretario Técnico

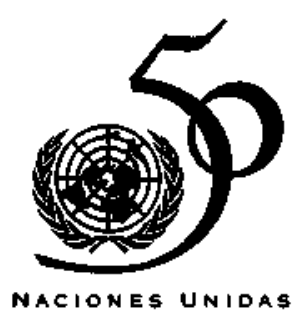


Democracla y desarrollo

Fernando H. Cardoso

¿Es posible crecer con equidad?

Joseph Ramos

Estabilidad y estructura: Interacclones en el crecimiento económlco

Jose Maria Fanelli y Roberto Frenkel

Reforma a los sistemas de pensiones en América Latina

Andras Uthoff

Tendenclas económicas en China: significado para el comerclo con América Latina y el Caribe

Mikio Kuwayama

El Intercamblo económico entre América Latina y las

economias dinámicas de Asla

Ronald Sprout

La relación económica entre la Amórica Latina y la Unlón Europea

Roberto Smith Perera

Nuevas implicaclones de las reglas do orlgen

Eduardo Gitli

Globalización y reestructuración energética en Amórlca Latina

Fernando Sánchez Albavera

El caloidoscoplo de la competitlvidad

Geraldo Muller

La privatización de los servicios públicos del agua

Miguel Solanes

¿Cuénto se puede gastar en educaclón?

Guillermo Labarca

Mujeres y migranteo: deslgualdades en el

mercado laboral de Santlago de Chile

Ivonne Szasz

Orlentaciones para los colaboradores de la Rovista de la CEPAL 


\section{¿Cuánto se puede gastar en educación?}

\section{Gulliermo Labarca}

Consultor,

División de Desarrollo

Productivo y Empresarial, CEPAL
Los sistemas educativos de América Latina emplean una tecnología adoptada de la que generaron los países actualmente más industrializados. Esta tecnología hace uso intensivo del trabajo, concentrando el gasto en los salarios del personal do. cente y administrativo, y su utilización eficiente - tal como se la aplica en los países industrializados - conlleva un elevado gasto por alumno. Siguiendo recomendaciones de organismos internacionales, muchos gobiernos de la región han manifestado la intención de aumentar el gasto actual en educación a entre $6 \%$ y $8 \%$ del producto interno bruto. Pero en los países de América Latina los recursos necesarios para lograr un sistema eficiente de cobertura universal superan con mucho esta cifra. La educación pública no tiene mecanismos para acceder a recursos suficientes; sólo las escuelas privadas que reciben apoyo financiero de las familias de los estudiantes están en condiciones de emplear adecuadamente las tecnologías seffaladas. Para resolver el problema educativo en la región es necesario elaborar una política que incentive el desarrollo de tecnologías educativas más eficientes, en general con uso más intensivo de capital, tomando como punto de partida las experiencias exitosas de la región. 


\section{I}

\section{Introducción}

Pocas veces en la historia de los sistemas educativos en América Latina ha habido tanta coincidencia entre los distintos actores sociales sobre lo que es y lo que idealmente debiera ser la educación escolar. Gobiernos, educadores, académicos, empresarios, trabajadores, iglesias, agrupaciones culturales y otras atribuyen objetivos similares a la educación y lo hacen en un lenguaje muy parecido. La mayoría de los análisis muestran una expansión de la cobertura educativa, en todos sus niveles, junto con una declinante calidad de la enseî́anza. Se verifica además que la educación formal no responde adecuadamente a las demandas presentes y futuras del aparato productivo y que no contribuye considerablemente a mejorar la equidad social. Los análisis coinciden también al identificar cuáles son los más importantes desafíos a que se ve enfrentada la educación.

Entre ellos, dos se mencionan recurrentemente: mejorar la calidad, con las necesarias adecuaciones curriculares y didácticas a los avances de la ciencia, la tecnología y la pedagogía; establecer una relación más fluida con el mercado del trabajo y con los niveles superiores de la educación, incluyendo aquí la incorporación de las principales transformaciones técnicas actuales y las que es razonable esperar que ocurran en el futuro próximo; crear condiciones educativas que contribuyan a transformar las estructuras productivas de la región y amplíen la equidad social; privilegiar una enseñanza centrada en el desarrollo de las habilidades de los individuos, abandonando pedagogías orientadas principalmente a la adquisición de conocimientos; formar valores y principios éticos que ayuden a lograr un buen desempeño en los diferentes ámbitos de la vida social, y dar acceso universal a los códigos de la modernidad, lo que obliga, entre otras cosas, a mejorar la enseñanza de las ciencias y la tecnología (CEPAL, 1990).

También se observan ciertas coincidencias acerca de cuáles son las estrategias más apropiadas para

\footnotetext{
$\square$ Las observaciones y comentarios que hicieron Joseph Ramos y Jorge Katz a una primera versión de este trabajo permitieron a este autor precisar conceptos, afinar argumentos y organizar mejor la información. Toda imprecisión o confusión que aún quede es de su exclusiva responsabilidad.
}

enfrentar estos desafíos, o al menos de cuáles son las condiciones que los países deben reunir para avanzar hacia los objetivos propuestos. Las que parecen concitar más atención son: la descentralización del sistema educativo y una mayor autonomía curricular y de gestión de los establecimientos; una regulación que permita coordinar las distintas unidades del sistema de educación entre sí y con el entorno económico, social y político; políticas de consenso entre todos los sectores involucrados, y acceso universal a la educación media o secundaria, en el supuesto de que la primaria ha alcanzado ya cobertura universal (CEPAL, 1990).

Pero, como es sabido, no basta con coincidir en los principios y en los pasos que han de darse para que las políticas sean eficaces. En la formulación y aplicación de estrategias se advierten rigideces debidas generalmente a contradicciones con políticas macroeconómicas y de equilibrio fiscal; a carencias infraestructurales de los sistemas de educación; a escasez de recursos humanos calificados, especialmente en la educación técnica y profesional; a demandas, bien o mal fundadas, de los sindicatos de maestros; al desconocimiento de las tecnologías educativas más apropiadas; a un apoyo muy tímido de instituciones sociales, entre otras las empresas, cuando éste es necesario para implementar determinados planes; y a la ausencia de un entorno cultural apropiado.

Pero el obstáculo más importante -y ésta constituye la afirmación central del presente artículo- es la imposibilidad de aplicar los modelos educativos implícitos o explícitos en las propuestas de mejoramiento de la calidad de la educación latinoamericana, por las restricciones financieras y culturales que impone el entorno.

Para fundamentar esta afirmación se describirán los modelos educativos y la tecnología implícita en ellos; enseguida se mostrará la distribución del gasto en educación en países industrializados y en América Latina; se examinara la manera en que este modelo educativo se aplica en la región; se sugerirán algunas opciones tecnológicas para la educación, y se expondrán sus efectos en la política educativa. 


\section{II}

\section{Los modelos de referencia}

Los modelos educativos de la región están inspirados por el Liceo francés, el Gimnasio alemán y las escuelas "comprensivas" de Estados Unidos, Suecia o Inglaterra, que en mayor o menor medida han venido inspirando los sistemas educativos regionales por lo menos desde inicios de este siglo. Las escuelas primarias también han sufrido esa influencia, siendo en muchos casos una versión diluida de la escuela secundaria. Tales modelos han contribuido a determinar los objetivos de las escuelas, los contenidos de la enseñanza y la tecnología educativa empleada en las modalidades de educación general. También la noción de diferenciación entre la educación general científica humanista y la educación técnica proviene de los mismos modelos organizativos y pedagógicos que se han impuesto en América Latina. No se desconoce aquí que en la región lo que ha habido son adaptaciones locales, pero éstas no han sido tan profundas como para cambiar las dimensiones esenciales de los modelos (MECE 1.2, 1993).

Las reformas educativas que se proponen y en parte se llevan a cabo hoy en América Latina corresponden a adaptaciones de esos modelos a las necesidades economicas y sociales de hoy. Este proceso ya se ha efectuado, o se está efectuando, en muchos de los países industrializados, casi siempre siguiendo los principios organizativos y las tecnologías pedagogicas del modelo original. Chile, Colombia, Jamaica y México, entre otros, han emprendido reformas educativas con estas características.

Desde el ángulo de la tecnología educativa preocupación central de este artículo-, los sistemas educativos que sirven de modelo a la región están articulados en torno al libro como medio didáctico, y a la ciencia positiva como contenido y método curriculares. Las bases tecnologicas de estos sistemas fueron establecidas a principios del siglo XIX en Europa, y atribuyeron funciones específicas a los docentes y al material de enseñanza. El modelo educativo norteamericano - la escuela comprensiva, que posteriormente fue adoptada con modificaciones mayores o menores en el Reino Unido, Japon y los países escandinavos-, si bien altera aspectos de la estructura de los sistemas educativos secundarios, no introduce modificaciones esenciales en la tecno- logía educativa de los sistemas europeos (Johnson y Packer, 1987).

Los libros suponen la existencia de un determinado contexto cultural y de una serie de instituciones que, directa o indirectamente, apoyan la labor de la escuela. Entre otras hay que mencionar las bibliotecas escolares, comunales o de barrio que ponen los libros al alcance de los estudiantes; los museos de arte, tecnológicos o científicos en las ciudades, que complementan e ilustran conceptos e informaciones aprendidos en la sala de clases; una prensa escrita o televisiva con referencias y análisis que plantean problemas y entregan informaciones, las que posteriormente pueden ser integradas a la práctica escolar; y en algunos casos, en especial en Alemania y tímidamente en otros países, una relación con el mundo productivo que facilita la tarea de las escuelas técnicas y profesionales (Johnson y Lundvall, 1988).

El libro, en estas diferentes modalidades educativas, es el depositario del conocimiento y cada docente un iniciador y comentarista de las disciplinas científicas particulares. El docente es por lo tanto el otro componente esencial de la "tecnología educativa" I de estos sistemas escolares; se distingue más por su especialidad en alguna de las áreas del conocimiento que por sus dotes pedagógicas; su papel en la difusión del conocimiento es, entre otros, el de ayudar a los estudiantes a delimitar el campo disciplinario y a organizar la información pertinente; iniciar y orientar a los estudiantes en el proceso de búsqueda de información, e informarles sobre los avances de la ciencia. En esta tecnología educativa el conocimiento está depositado en textos, pero es activado por el docente, es decir, por un recurso humano altamente especializado y calificado. El docente debe tener conocimientos disciplinarios avanzados y capacidad de responder a interrogantes y dudas de los estudiantes, y debe organizar su trabajo de manera que le permita generar incentivos que estructuren la actividad de los escolares, y seguir y evaluar los avances de éstos.

Las labores del docente se distribuyen entre ho-

\footnotetext{
1 Tecnología en el sentido de combinación de recursos materiales y humanos. En la educación el recurso humano decisivo para cualquier cálculo económico es el docente.
} 
ras de clase efectivas, lectura y preparación de las clases, atención individual de los estudiantes, control y corrección de exámenes y pruebas, y trabajo administrativo y de gestión del establecimiento. El tiempo dedicado a cada una de estas tareas fluctúa en los distintos sistemas educativos nacionales, pero en promedio dedica a clases entre 35 y $50 \%$ del tiempo de trabajo; entre 20 y $33 \%$ a la preparación de clases, la lectura para mantenerse al día en los avances de la pedagogía y de las disciplinas científicas, la corrección de pruebas y la atención de alumnos,y el tiempo restante a tareas administrativas, de perfeccionamiento y otras.

Originalmente, esta forma de organizar la educación -en el siglo XIX en Europa y XX en Estados Unidos y Japón- apuntaba a proveer a la sociedad de líderes y funcionarios públicos de alta categoría. Después de la segunda guerra mundial se amplí la cobertura del sistema educativo en esos países para acompanar los procesos de democratización y de reorganización productiva. Esto elevó considerablemente el gasto en educación. En promedio, entre 1950 y 1960 el porcentaje del PIB que los países de la Organización de Cooperación y Desarrollo Económicos (OCDE) destinaron a educación subió del 3 al 5\% y en torno a estas cifras se ha movido en los años posteriores. La casi totalidad de este gasto se hizo y se sigue haciendo con fondos públicos (OCDE, 1992).

En las últimas décadas se ha modificado el papel de la educación en estas sociedades, planteando nuevos problemas y generando movimientos de reforma educativa casi permanentes. Algunas de estas modificaciones, especialmente las que vinculan más estrechamente el mundo del trabajo con el de la educación, han sido conocidas en la región, y se intenta aplicarlas. El sistema dual alemán ha sido el que probablemente ha llamado más poderosamente la atención, entre otras cosas porque modifica tanto el papel y la función del docente, como su relación con el conocimiento y el proceso para su formación y perfeccionamiento. Esta innovación permanece restringida a escuelas técnico-profesionales.

En las otras modalidades sigue predominando, de hecho o como referencia, la tecnología educativa clásica, a pesar de las modificaciones pedagogicas y de gestión de las escuelas. La "función de producción educativa", es decir la combinación de recursos humanos y materiales usados para proveer de servicio educativo a la población, no es en esencia diferente de la que se implanto en el siglo XIX e inicios del XX en los países industrializados. Aun cuando ha habido modificaciones pedagógicas y didácticas en la educación escolar, desde un punto de vista económico no ha habido progreso técnico. Más aún, se puede afirmar que los sistemas educativos tienden a ser cada vez más ineficientes, ya que las sustituciones de insumos para escolarizar a cada individuo no han disminuido los costos para obtener un resultado comparable, sino que los han aumentado.

En el gráfico 1 se muestra la función de producción de las modalidades más habituales de la educación. Sobre el segmento $\mathrm{AB}$ de la curva $\mathrm{E}$ se sitúan las combinaciones existentes. Todas ellas hacen uso intensivo de trabajo (T). Las combinaciones más a la izquierda de $\mathrm{C}$ (con uso más intensivo de capital) son sólo teóricas, no tienen expresiones reales. Algunas de las innovaciones introducidas en los años setenta y ochenta desplazan la curva hacia $\mathrm{E}^{\prime}$ y son entonces más ineficientes; además, se mantienen en el segmento A'B'. EI problema económico es encontrar una curva que se desplace hacia $E^{\prime \prime}$.

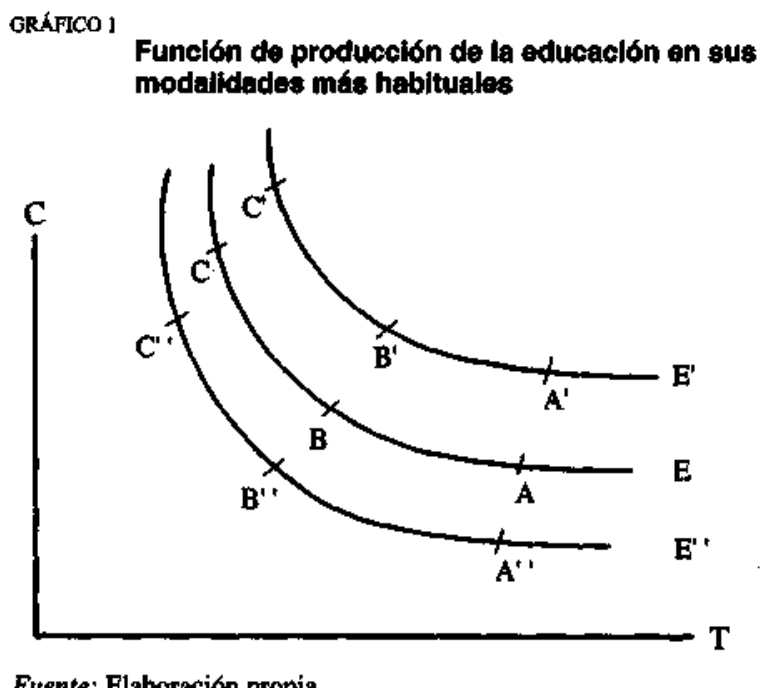

Fuente: Elaboracion propia. 


\section{III}

\section{El gasto en la educación escolar}

La aplicación de estas tecnologías está asociada a determinados costos. En los países industrializados estos costos son los que se indican en el cuadro 1 .
El gasto real por alumno refleja mejor los costos de los servicios educacionales que otros indicadores que se usan habitualmente, como la proporción del

CUADRO 1

OCDE: Gasto púbilco anual en educaclón en algunos paises (Promedio en 1970-1988)

\begin{tabular}{|c|c|c|c|c|}
\hline & $\begin{array}{l}\text { Como \% del } \\
\text { PIB (nominal) }\end{array}$ & $\begin{array}{l}\text { Por alumno, } \\
\text { norminal } \\
\text { (dollares) }\end{array}$ & $\begin{array}{l}\text { Por alumno, } \\
\text { real } \\
\text { (dolares) }\end{array}$ & $\begin{array}{l}\text { Por alumno, } \\
\text { nominal, } \\
\text { (\% del PIB per } \\
\text { cápita) }\end{array}$ \\
\hline Australia & 5.4 & 2693 & 3431 & 25.4 \\
\hline Canadá & 6.9 & 4013 & 4106 & 30.5 \\
\hline Estados Unidos & 4.6 & 3520 & 3418 & 21.2 \\
\hline Francia & $5.6^{\circ}$ & 2254 & 2651 & $\ldots$ \\
\hline Alemania & 4.1 & 2039 & 2230 & 20.0 \\
\hline Italia & 5.0 & 1783 & 3284 & 23.9 \\
\hline Japón & 4.7 & 2344 & 2324 & 21.4 \\
\hline Paises Bajos & 6.6 & 2567 & 3158 & 29.5 \\
\hline Suecia & 7.0 & 4197 & 4358 & 34.8 \\
\hline Noruega & 5.6 & 3657 & 3751 & 26.1 \\
\hline Reino Unido & 4.9 & 1758 & 2463 & 21.9 \\
\hline
\end{tabular}

Fuente: Datos de OCDE, 1992.

a Porcentaje del PIB real.

producto destinada a educación. El gasto real tiene en cuenta las situaciones nacionales en lo que se refiere al poder comprador de educacion. Las diferencias entre países sefialadas en el cuadro 1 se deben en parte a diferencias salariales de los docentes (más altos en Suecia y en los Países Bajos) y del personal administrativo (Estados Unidos) y a una mejor relación costo-eficacia (Japón). Pero en todos los casos los medios didácticos, la manera de conservar y transmitir el conocimiento y la relación entre docente y medios didácticos es muy similar. Esto refleja distintas formas de aplicación de la tecnología, cuyos costos están afectados por condiciones locales.

Algunos organismos internacionales que se han ocupado del tema, entre ellos el Banco Mundial, la CEPAL y la UNESCo, han sugerido que el gasto en educación en los países en desarrollo se acerque a los niveles de gasto de los países industrializados. En términos agregados, esto significaría llegar a alrededor de $6 \%$ del producto.

El cuadro 2, que recoge cifras de algunos países de la región, muestra un gasto en educación que, salvo en Panamá y Jamaica, está lejos de las recomendaciones de los organismos internacionales. El nivel de los recursos destinados a educación se refleja, en primer lugar, en el gasto por estudiante, que en la región es alrededor de diez veces menor que en los países industrializados. Esta diferencia no se explica sólo por el nivel de desarrollo, sino también porque los países de la región gastan una proporción menor del producto en educación, y sobre todo, porque reducen los costos por diversos procedimientos.

Llama la atención también la distribución del gasto, que en la mayoría de los países incluidos se concentra en el gasto corriente; sólo Jamaica y México destinan una cantidad de recursos importantes a capital. Esta manera de distribuir los recursos contribuye a definir la manera de aplicar esta tecnología educativa en la región.

La tecnología educativa a que nos estamos refiriendo, cualquiera sea el país en que se la aplique, hace uso muy intensivo de mano de obra calificada. 


\begin{tabular}{|c|c|c|c|c|}
\hline & $\begin{array}{l}\text { Total, como } \\
\text { \% del PIB }\end{array}$ & $\begin{array}{l}\text { Gasto co- } \\
\text { miente co- } \\
\text { mo \% del } \\
\text { gasto total }\end{array}$ & $\begin{array}{l}\% \text { del gasto } \\
\text { corriente en } \\
\text { educación } \\
\text { secundaria }\end{array}$ & $\begin{array}{l}\text { Gasto por alumno } \\
\text { secundario, } \\
\text { nominal } \\
\text { (dolares del } \\
\text { affo) }\end{array}$ \\
\hline Argentina & 1.5 & 96.0 & 44.9 & 349.20 \\
\hline Bolivia & 2.4 & 99.5 & 13.5 & 61.70 \\
\hline Brasil (1988) & 3.9 & $\ldots$ & 7.2 & 248.40 \\
\hline Chile & 3.4 & 97.5 & 17.3 & 177.30 \\
\hline Colombia (1989) & 2.9 & 95.2 & 27.6 & 126.80 \\
\hline Costa Rica & 4.6 & 96.6 & 17.1 & 317.30 \\
\hline Ecuador & 2.7 & 94.7 & 32.0 & 100.30 \\
\hline Hait'́ & 1.8 & 99.9 & 19.1 & 57.50 \\
\hline Jamaica & 5.9 & 86.7 & 33.2 & 251.20 \\
\hline México & 4.1 & 61.2 & 29.2 & 257.30 \\
\hline Panamá & 5.7 & 97.3 & 23.3 & 295.90 \\
\hline Perú (1987) & 3.5 & 94.6 & 20.4 & 187.00 \\
\hline
\end{tabular}

Fuente: UNESCO, 1994.

Casi todos los países cuyos sistemas educativos tienen esta característica gastan más del $60 \%$ de los recursos públicos destinados a educación en salarios. Algunos, como Alemania, Canadá, Francia, el Reino Unido y la República de Corea, gastan en este rubro más del $70 \%$; otros, entre los que se encuentran Argentina, Bélgica, Chile y Costa Rica, gastan más del $80 \%$, y países como Bolivia y Colombia, más del $90 \%$.

El gasto en capital es de $5 \%$ o menos del gasto total en los países de la región, salvo en Jamaica y México, que destinan a inversiones 13.3 y $38.8 \%$ del gasto (UNESCO, 1993). En los países de la OCDE el gasto tiene habitualmente un componente mayor de capital (alrededor del 10\% del gasto total en educación en los años noventa).

Los datos agregados del gasto en educación (corriente y de capital) no reflejan claramente las diferencias entre los paŕses industrializados y los de América Latina. Si bien en los países de la OCDE la tasa de crecimiento del gasto corriente ha sido mayor que la del gasto de capital a partir de 1970, ese crecimiento se basó en inversiones ya realizadas en el período anterior. La participación de las inversiones de capital ha disminuido en Australia de 19.3 a $7.2 \%$, en Austria de 20.0 a $8.5 \%$, en Alemania de 20.8 a $7.5 \%$, en Holanda de 18.0 a $5.9 \%$, en Noruega de 16.1 a $6.5 \%$, en Suiza de 22.1 a $10.5 \%$, etc. (OCDE, 1992). La elasticidad del crecimiento del gasto corriente respecto del gasto total en educación es mayor que uno en los años setenta y ochenta. La participación de las inversiones de capital subió posteriormente en los noventa, llegando al $10 \%$ del gasto público en educación.

Estas variaciones reflejan coyunturas políticas y sociales de los países de la OCDE que asocian el crecimiento del gasto corriente con la expansión de la matrícula del sistema y no con cambios importantes en las tecnologías educativas empleadas. El gran gasto en edificios y material de enseñanza (bibliotecas, laboratorios, etc.) ya se realizó en los años sesenta y principios de los setenta. Ahora se trata sobre todo salvo probablemente en el Reino Unido (excluida Escocia), Australia y los países escandinavos- de perfeccionamientos de modalidades ya establecidas. Por lo demás, las innovaciones pedagógicas más importantes (es decir, las distintas formas de escuelas comprensivas), no operan con tecnologías pedagógicas radicalmente diferentes. Son innovaciones curriculares y estructurales que no alteran esencialmente la relación entre los medios didácticos y el docente, aun cuando permitan aumentar la cantidad de estudiantes por docente al suprimir la diferenciación entre distintas modalidades escolares.

En América Latina, en cambio, ha habido una falta crónica de inversión en infraestructura física y material de enseñanza. Esta situación, que se ha venido arrastrando en las últimas décadas, ha permitido operar con costos más bajos, pero con deterioro de la calidad de los servicios educativos públicos. 


\section{IV}

\section{Las adaptaciones del modelo}

\section{a la realidad de América Latina}

Las grandes diferencias entre los países industrializados y los de America Latina resultan de una "adaptación tecnológica" que se hace en algunos países de la región. Esta consiste en una utilización casi exclusiva del tiempo de trabajo del docente en hacer clases, suprimiendo o disminuyendo al mínimo los tiempos para leer, preparar clases, atender a estudiantes, etc. Como resultado, los sistemas educativos terminan aplicando tecnologías que son más cercanas a las de antes del uso generalizado del libro impreso, vale decir, al tipo de escuela organizada en los conventos medievales. En ella el docente es el depositario del conocimiento, y la técnica más usada para la difusión de conceptos e información es el dictado. El docente es un reproductor $y$, a veces un comentarista, de sentencias cuyo aprendizaje memorístico es el objetivo de la práctica escolar para los estudiantes. El control de conocimientos se realiza sobre los dictados del docente, y los libros, sin desaparecer, no tienen un papel relevante.

Detrós de esta adaptación hay, sobre todo, razones económicas. En cuarenta horas de clases semanales un docente puede hacer alrededor de 2.5 veces más lecciones que en las escuelas que originaron el modelo. A esto se agrega que los cursos son más grandes, sin que eso genere entrabamientos: como esta manera de enseñar no estimula la participacion estudiantil, importa menos la cantidad de escolares en la sala de clases. Disminuye así la relación docente/estudiante y por lo tanto mejora la relación costo/efectividad (medida en horas de clase impartida por alumno), lo que no significa necesariamente una educación de mejor calidad.

Al observar la relación estudiante/docente en diferentes países a fines de los años ochenta (cuadro 3), vemos que los países industrializados con sistemas educativos de estructura organizativa clásica, es decir, con modalidades diferenciadas, mostraban en general un número menor de escolares por docente que aquéllos en los que las escuelas comprensivas eran las más numerosas, y también menor que en los parses de América Latina y el Caribe. ${ }^{2}$

\footnotetext{
2 Estas cifras entregan solo una aproximación porque están contabilizados todos los docentes, cualquiera sea la extensión de su jomada de trabajo, haciendo equivalentes a los que trabajan 40
}

\begin{tabular}{|c|c|c|}
\hline \multicolumn{3}{|l|}{ UADRO 3} \\
\hline & $\begin{array}{l}\text { En la educación } \\
\text { secundaria } \\
\text { total }\end{array}$ & $\begin{array}{c}\text { En la educación } \\
\text { vocacional }\end{array}$ \\
\hline \multicolumn{3}{|l|}{$\begin{array}{l}\text { Sistemas escolares con } \\
\text { diferentes modalidades } \\
\text { (escuela clasica) }\end{array}$} \\
\hline $\begin{array}{l}\text { Bélgica (1987) } \\
\text { Austria (1989) } \\
\text { Rep. Dem. Alemana (1988) } \\
\text { Italia (1989) } \\
\text { Checoslovaquia (1989) } \\
\text { Francia (1989) } \\
\text { Alemania, Rep. Federal de (1988) }\end{array}$ & $\begin{array}{r}7.03 \\
8.45 \\
8.96 \\
9.18 \\
9.86 \\
12.42 \\
14.02\end{array}$ & $\begin{array}{r}9.00 \\
6.75 \\
9.13 \\
7.93 \\
17.24 \\
22.26\end{array}$ \\
\hline \multicolumn{3}{|l|}{$\begin{array}{l}\text { Sistemas escolares con } \\
\text { escuelas comprensivas }\end{array}$} \\
\hline $\begin{array}{l}\text { Australia (1990) } \\
\text { Estados Unidos (1986) } \\
\text { Japón (1989) } \\
\text { Singapur (1980) } \\
\text { República de Corea (1990) }\end{array}$ & $\begin{array}{l}12.60 \\
13.35 \\
17.10 \\
19.45 \\
25.23\end{array}$ & $\begin{array}{r}\cdots \\
\cdots \\
9.18 \\
21.95\end{array}$ \\
\hline $\begin{array}{l}\text { America Latina y el Caribe } \\
\text { Ecuador (1987) } \\
\text { Brasil (1989) } \\
\text { México (1990) } \\
\text { Venezuela (1988) } \\
\text { Panama (1988) } \\
\text { Colombia (1989) } \\
\text { Perú (1990) } \\
\text { Bolivia (1989) } \\
\text { Jamaica (1988) }\end{array}$ & $\begin{array}{l}14.41 \\
14.42 \\
16.66 \\
17.27 \\
19.41 \\
19.88 \\
20.92 \\
21.68 \\
26.20\end{array}$ & $\begin{array}{r}15.49 \\
\ldots \\
13.62 \\
\ldots \\
18.06 \\
18.00 \\
\ldots \\
\ldots \\
16.49\end{array}$ \\
\hline
\end{tabular}

Fuente: Elaborado con datos de CEPAL, 1993.

Sólo la ex República Federal de Alemania y Francia exhibían una relación entre alumnos y docente que los asemejaba a países como Brasil o Ecuador. Esto se explica, en el caso de la educación vocacional alemana, por las características especiales de la escuela dual, que divide la enseñanza entre

horas semanales con los que solo trabajan pocas horas. En los paises en los que son más habituales los contratos de jomada parcial, la cantidad de docentes está abultada en relación al número de estudiantes. 
docentes en las escuelas y trabajadores-tutores en las industrias, aumentando así la cantidad de estudiantes que atiende cada docente en las escuelas. Además, el peso de la educación vocacional en ambos países es considerable, lo que afecta la relación total entre escolares y docente indicada en la primera columna del cuadro 3. En ambos países europeos la relación alumnos/ docente en la educación secundaria, excluida la vocacional, era de 11.3. En la ex República Democrática Alemana era de 10.1, en Italia de 9.20 y en Austria de 8.27. Por otra parte, hay que tener en cuenta que en todos los países europeos con sistemas de educación secundaria general muy diferenciados se advierten disparidades cualitativas entre las distintas orientaciones; aquellas que preparan para la educación superior tienen una relación alumnos/docente más favorable que las escuelas terminales.

Entre las modificaciones que se han introducido en América Latina para adaptar las tecnologías educativas y reducir costos se halla precisamente la de la relación alumnos/docente. Otra adaptación importante ha sido la de disminuir las horas de clase por alumno. Así, en la región los estudiantes asisten en las escuelas públicas a entre 700 y 900 horas de clase al año, mientras que en Europa esta cifra se sitúa cerca de las 1200 horas anuales y en la República de Corea y Japón se aproxima a las 1400 . En numerosas publicaciones aparecidas en países de la OCDE, en especial Estados Uni- dos, se estima necesario que los países industrializados alcancen la cifra de Japon para superar las deficiencias que se observan en sus sistemas educativos.

Todas las adaptaciones que se efectúan en América Latina van unidas a bajos salarios para los docentes. Si se acepta que hay una correspondencia entre el nivel del salario y la calidad técnica del asalariado y, por lo tanto, que los salarios más altos atraen a los profesionales más competentes, el sistema de educación latinoamericano estaría empleando un personal de calidad inferior sin posibilidades reales de mejorar su calificación mediante capacitaciones posteriores o estímulos al perfeccionamiento. De existir tales estímulos, ellos no se aprovechan efectivamente porque el peso de la labor propiamente docente no deja a ese personal el tiempo necesario.

El problema de la calidad es crucial en la actividad educativa. Los países industrializados la buscan mediante la formación básica de los maestros y posteriores períodos de capacitación y de actualización de conocimientos. Tanto la formación básica como la capacitación posterior son componentes importantes de este modelo pedagogico.

Aun cuando no se dispone de mediciones similares para la educación secundaria, hay elementos para suponer que la brecha entre los páses más eficientes y los menos eficientes se agranda considerablemente, y que esto afecta especialmente a Estados Unidos y los países latinoamericanos.

\section{$\mathrm{V}$}

\section{El modelo educativo en las condiciones locales}

Las políticas educativas de varios parses de la región apuntan a redefinir el modelo educativo y al mismo tiempo corregir los efectos adversos de la situación actual sobre el proceso de aprendizaje. La necesidad de hacerlo se ha planteado con más frecuencia por razones externas a la educacion, entre otras, por la imposibilidad de que las escuelas respondan adecuadamente a la demanda presente de recursos humanos y a la previsible en el futuro próximo, y también por la demanda de cambios en los sistemas de educación generada por las exigencias crecientes de equidad y democratización (MECE 1.2, 1993).

Cabe preguntarse en este momento si es posible estructurar nuevos sistemas educativos o mejorar los existentes para que puedan responder a lo que se es- pera de ellos, aplicando tecnologías pedagogicas inspiradas en la escuela clásica y con un nivel de gasto del 6 o $7 \%$ del producto. La pregunta es importante, por cuanto la demanda de educación es muy elástica y tiende a aumentar más rápidamente que la oferta, afectando de inmediato la calidad del servicio. Además, dadas las características del "bien educacion", que es a la vez un producto de consumo y una inversión, su demanda no está regulada por los mecanismos de mercado. Es decir, las variaciones de los precios que pagan los usuarios de la educación no van a afectar directamente la demanda total, si ellos tienen la posibilidad de acceder a este bien sin costo o con un costo muy bajo (educación pública).

Por lo demás, los incrementos del gasto en edu- 
cación no siempre mejoran la calidad de los servicios educativos. Trabajos antiguos de Coleman (1966) y de Jencks (1972) sobre la efectividad de estos incrementos mostraron que el gasto marginal en material escolar e infraestructura tiene una escasa relación con el logro escolar. Investigaciones posteriores en los países de la OCDE sobre las funciones de producción educativa concluyeron que no hay una relación sistemática entre el gasto en educación y el rendimiento de los estudiantes (OCDE, 1992). En los países industrializados algunos autores han planteado serias dudas sobre si los aumentos adicionales del gasto $-\mathrm{y}$ en qué medida - son la mejor manera de elevar la efectividad de las escuelas. ${ }^{3}$

Para definir la o las modalidades educativas más apropiadas para la región es preciso determinar en primer lugar la estructura de costos mínima de la tecnología educativa que sirve de referencia, y en segundo lugar, compararla con el gasto que los diferentes países están en condiciones y en disposición de hacer. No se pretende sugerir con esto que las consideraciones presupuestarias son las únicas que deben tomarse en cuenta al formular políticas, sino sólo que es preciso tenerlas en cuenta.

Los países industrializados aplican distintas versiones del mismo modelo. Cada uno de ellos ha hecho sus propias adaptaciones. Japón y el Reino Unido gastan mucho menos, en términos reales, que Canadá o Suecia. La República de Corea tiene una relación alumnos/docente más parecida a la de Jamaica o Bolivia, y Japón a la de México o Venezuela. Sin embargo, la calidad de la educación es muy superior en los países asiáticos que en los latinoamericanos y, según indicadores de rendimiento, que en los europeos y en Estados Unidos. La cuestión estriba en saber cuáles son los límites de la adaptación tecnológica, es decir, hasta dónde se puede modificar el modelo sin que el producto (la educación) pierda calidad (Duran-Drouhin, 1993; Ferreyra, 1993).

Las distintas variaciones del modelo educativo se pueden clasificar en tres agrupaciones principales: i) la educación diferenciada clásica, con varias modalidades; ii) la escuela comprensiva, y iii) la que se ha desarrollado en algunos países del Este asiático. La primera se encuentra en Alemania, Francia, Italia, los

\footnotetext{
3 Si se uviera la información y los medios para estimar adecuadamente los beneficios de la educación (economicos, sociales, educativos, etc.) se podria determinar con precisión la rentabilidad de las inversiones en este sector. La información disponible permite adelantar la hipótesis de que el beneficio marginal del gasto anual por sobre 2000 dólares al año por estudiante tiende a cero.
}

Países Bajos y otros. La segunda en Estados Unidos, Australia, el Reino Unido y los países escandinavos, entre otros, y la tercera, en Japón, la República de Corea y Singapur.

Los sistemas comprensivos tienen un número de alumnos por docente más alto que los sistemas con varias modalidades, pero no operan necesariamente con un gasto menor. Suecia y Noruega, que han organizado toda su educación secundaria en escuelas comprensivas, están entre los países que más gastan en educación, cualquiera sea el indicador que se elija.

Los países del Este asiático, en particular Japón, la República de Corea y Singapur, también han organizado sus sistemas educativos en escuelas comprensivas (Japón), o con algún grado de diferenciación (Malasia, la República de Corea o Singapur). Estos sistemas muestran un alto rendimiento académico y una alta eficacia, en tanto que el gasto como función del producto y el gasto total por alumno son más bajos que los de los países industrializados. La eficiencia académica de esos sistemas se explica por el papel de la escuela en la asignación de roles laborales, la importancia de los diplomas escolares y, sobre todo, por el régimen disciplinario con que operan los establecimientos educativos. Finalmente, la relación de la escuela con la familia es muy estrecha, tanto en lo académico como en lo disciplinario. Estas dimensiones de la educación están enmarcadas y definidas por las culturas de esos países.

Pareciera que la región no puede optar por este modelo educativo tal como se ha desarrollado en los países del Este asiático, aun cuando ofrece ventajas comparativas innegables en materia de costos. La disciplina y los incentivos éticos han ido perdiendo eficacia en la educación latinoamericana. El apoyo y complementación entre el hogar y la escuela es otro aspecto cuya deficiencia en la región atenta contra la posibilidad de organizar la práctica escolar con estrategias inspiradas en la experiencia asiática.

Más pertinente es la información que proviene de los sistemas europeo, australiano o estadounidense. Tomando éstos como referencia, a continuación se definen algunos de los requerimientos indispensables para aplicar con eficacia esta tecnología educativa.

i) El número de estudiantes por docente debe ser de alrededor de 10.

ii) Los estudiantes deben asistir a $\mathbf{1 2 0 0}$ horas de clase al año.

iii) La cobertura de la educación, por lo menos hasta el décimo año, debe ser del $100 \%$.

iv) La calidad de los docentes, medida por su 
salario anual, debe alcanzar niveles comparables al de otros profesionales. Para ello se puede utilizar una de las dos estimaciones siguientes: en la primera el salario de los docentes se estima sobre la base del salario medio de los profesionales de la administración pública en 1990; en la segunda se estima sobre la base del salario inicial profesional de la administración pública. ${ }^{4}$ v) la infraestructura, en especial los edificios, debe crecer de manera de albergar las extensiones de tiempo, de clases y matrícula que resulten de las medidas anteriores.

vi) Los establecimientos deben contar con bibliotecas adecuadas.

Estos requerimientos afectan de manera diferente a los distintos países de la región (cuadro 4). educatlva clásica en algunos países

\begin{tabular}{|c|c|c|c|}
\hline & $\begin{array}{l}\text { Dotación } \\
\text { adicional de } \\
\text { docentes } \\
\text { del nivel se- } \\
\text { cundario } \\
\text { (1) }\end{array}$ & $\begin{array}{l}\text { Dotacion } \\
\text { adicional de } \\
\text { docentes del } \\
\text { nivel secun- } \\
\text { darjob } \\
\text { (2) }\end{array}$ & $\begin{array}{c}1+2 \text { como } \% \\
\text { de la dota- } \\
\text { cjon de docentes del } \\
\text { nivel secun- } \\
\text { dario } \\
\text { (3) }\end{array}$ \\
\hline $\begin{array}{l}\text { Bolivia } \\
\text { Brasil } \\
\text { Chile } \\
\text { Colombia } \\
\text { Ecuador } \\
\text { Jamaica } \\
\text { México } \\
\text { Panamá } \\
\text { Perú } \\
\text { Venezuela }\end{array}$ & $\begin{array}{r}11500 \\
95400 \\
12000 \\
114800 \\
21400 \\
14500 \\
241500 \\
8800 \\
83400 \\
42000\end{array}$ & $\begin{array}{r}13100 \\
178400 \\
9200 \\
15300 \\
54000 \\
7110 \\
280000 \\
5300 \\
38400 \\
16300\end{array}$ & $\begin{array}{r}259 \\
115 \\
72 \\
113 \\
141 \\
240 \\
130 \\
144 \\
146 \\
97\end{array}$ \\
\hline
\end{tabular}

Fuente: Elaboración propia con datos de UNESCo (1992) y Banco Mundial (1993).

a Estimada, considerando una relación de 1 a 10 entre docente y alumnos, 1200 horas de clases anuales, y manteniendo la cobertura actual.

- Estimada, considerando la incorporación a 10 años de educación escolar de la población en edad correspondiente que hoy no está matriculada, una relación 1 a 10 entre docente y alumnos, y 1200 horas de clases anuales.

Para mejorar la educación secundaria en América Latina, y ponerla en condiciones de responder a las exigencias que se le hacen, utilizando la organización escolar clásica, es preciso duplicar la cantidad de docentes en la mayoría de los parses, y en algunos de ellos, como Jamaica y Bolivia, sumar al contingente actual una cantidad equivalente a dos veces y media ese contingente, incurriendo en los costos com rrespondientes (cuadro 5).

El gasto corriente adicional en que incurririan los sistemas educativos si se atuvieran a estos parámetros serfa equivalente aproximadamente el $6.3 \%$ del PIB en Ecuador, $2.4 \%$ en Brasil, $3.3 \%$ en México, $5.8 \%$ en Venezuela, $6.2 \%$ en Colombia, $4.3 \%$ en Perú, $5.0 \%$ en Bolivia, $6.9 \%$ en Jamaica y $2.2 \%$ en Chile. La gravitación de tal gasto en la economía se aprecia al compararlo con el ingreso estatal como porcentaje del.PIB en 1991, que en Ecuador fue de $18.1 \%$, en México $14.7 \%$,

\footnotetext{
4 Se uso información de cinco parses y se extrapoló a los restantes.
}

en Venezuela $24.3 \%$, en Colombia $13.4 \%$, en Perú $8.3 \%$, en Bolivia $16.6 \%$ y en Jamaica y Chile cerca del $30 \%$ (Banco Mundial, 1993). Aun cuando la inversión en educación sea considerada rentable, no parece posible que los países destinen proporciones tan grandes del producto y del gasto estatal sólo a este rubro.

Al considerable aumento del gasto corriente que causana la aplicación cabal de esta tecnología, especialmente respecto de los salarios de los docentes, hay que sumar las erogaciones en edificios, bibliotecas y material de estudios que ella implica. No disponemos de información para estimar el gasto en infraestructura que sería necesario, pero si se consideran experiencias de los países industrializados, cabe colocarlo por sobre el $20 \%$ del gasto total en educación en períodos de reforma educativa, $y$ alrededor del $10 \%$ en períodos de estabilidad. Dadas las condiciones de la infraestructura de la región y su baja cobertura, el gasto en este rubro, especialmente edificios y bibliotecas, debería superar los porcentajes indicados. 

secundarla y costo salarial do aplicar la tecnología educatlva clásica

\begin{tabular}{|c|c|c|c|}
\hline & \multirow{2}{*}{$\begin{array}{l}\text { Gasto en sala- } \\
\text { rios de los docen- } \\
\text { tes secundarios } \\
\text { (millones de } \\
\text { de dolares en } \\
\text { los affos indicados) }\end{array}$} & \multicolumn{2}{|c|}{$\begin{array}{l}\text { Costo salarial de los docentes } \\
\text { secundarios, de aplicarse } \\
\text { la tecnología clasica } \\
\text { (millones de dólares de 1990) }\end{array}$} \\
\hline & & a & b \\
\hline Bolivia & $\ldots$ & 96.20 & 72.15 \\
\hline Brasil & $632.82(1986)$ & 5728.36 & 4296.30 \\
\hline Chile & $118.76(1990)$ & 701.42 & 526.07 \\
\hline Colombia & $114.71(1990)$ & 1408.87 & 1056.65 \\
\hline Ecuador & $62.73(1989)$ & 525.60 & 394.20 \\
\hline Jamaica & $41.14(1990)$ & 170.07 & 127.55 \\
\hline México & $1399.10(1990)$ & 12015.92 & 9011.94 \\
\hline Panamá & $44.93(1990)$ & 232.32 & 174.24 \\
\hline Perú & $147.87(1987)$ & 878.26 & 658.69 \\
\hline Venezuela & $80.57(1988)$ & 1033.63 & 775.22 \\
\hline
\end{tabular}

Fuente: Elaboración propia con datos de UNESCo (1992) y Banco Mundial (1993).

a Sobre la base de un salario anual de los docentes equivalente al salario profesional medio de la administración pública.

b Sobre la base de un salario anual de los docentes equivalente al salario inicial de la administración pública.

La conclusión más evidente es que la tecnología clásica no se puede masificar en la región: los recursos públicos son insuficientes para hacerlo, aunque hubiera una muy decidida voluntad política de privilegiar la educación. El uso exitoso de esta tecnología educativa que hacen algunas escuelas, cuyo rendimiento es comparable al de los establecimientos de países industrializados, no permite sostener que su aplicación pueda ser general. Se trata de colegios privados o públicos que cuentan con subvenciones especiales que equiparan sus ingresos a los de los países industrializados, pueden pagar salarios competitivos a los docentes y tienen una infraestructura adecuada. La mayor parte del gasto educativo privado de la región se concentra en este tipo de establecimientos y los beneficios que ellos otorgan son percibidos inmediatamente por quienes están dispuestos a efectuar este tipo de gasto. El gasto privado en educación escolar probablemente seguirá orien- tándose hacia las escuelas privadas y los beneficios seguirán quedando en el ámbito familiar, lo que no lo hace una alternativa de inversión plausible para todo el sistema escolar. ${ }^{5}$

Cabe tener en cuenta otra consideración: cuando se alcanza el nivel de eficiencia que tienen actualmente los sistemas educativos en los países industrializados, es muy difícil mejorarlo. ${ }^{6} \mathrm{~A}$ causa de su uso intensivo del recurso humano, la educación difícilmente puede mejorar su productividad. Por otra parte, hay obstáculos para modificar las tecnologías pedagógicas: los sistemas tienden a proteger el monopolio de los docentes en la transmisión del conocimiento, aun cuando la tecnología de la información se ha desarrollado muy rápidamente en los últimos veinte años y podría haber sido mucho más aprovechada. Esto se ha visto favorecido por las altas tasas de desempleo, que permiten seguir empleando maestros con muy bajas remuneraciones.

\footnotetext{
5 Existe hoy en la región una tendencia a atribuir a las empresas productivas responsabilidades de todo orden, entre ellas las de desarrollar las habilidades básicas, que tradicionalmente ha sido asumida por el sistema escolar público. Peres (1994) sugiere que si bien esto puede ser ineludible en la coyuntura actual, parece deberse menos a una virtud no intervencionista del Estado que a omisión en el cumplimiento de algunas de sus funciones básicas. Tenden-
}

cias similares se advierten en el área de la salud y en la del desamollo de sistemas nacionales de ciencia y tecnología.

6 Los conceptos de productividad y de eficiencia de la educación son difíciles de medir y a veces un tanto confusos, lo que lleva a que los responsables de las políticas pertinentes suelan dar más importancia y más presupuesto a algún aspecto en detrimento de orros, en vez de buscar la optimización de todos las "productos" de la educacion. 


\section{VI}

\section{Una opción posible: el cambio tecnológico}

Frente a este panorama, para lograr una educación concordante con las exigencias económicas y sociales del mundo contemporáneo y con los recursos disponibles o potenciales, conviene explorar otras alternativas. La estrategia que siguieron los paises industrializados, y en alguna medida también la República de Corea (en sus respectivos períodos de posguerra) no parece ser aplicable en la región: un aumento explosivo del gasto público en educación en períodos relativamente breves, multiplicando los montos asignados a educación entre 5 y 10 veces en menos de diez años.

La o las opciones de organización escolar en América Latina deben apoyarse en el supuesto de que el gasto en educación, especialmente el público, debe aumentar a niveles más altos de los que son habituales en ella. La cifra de 6 a $7 \%$ del PIB es una que la mayoría de los países de la región podría llegar a gastar, ${ }^{7}$ causando algún impacto focalizado. Pero aumentar así el gasto, conservando al mismo tiempo el modelo educativo, sólo permitirá correcciones graduales de índole general y una leve mejoría de los resultados. La coyuntura actual y la experiencia de constantes modificaciones marginales acumulada en la región reclama lo que Peres (1994) llama una "revolución estructural", ya que es evidente que las estrategias de política y gestión educativas aplicadas muestran sus límites.

Una de las líneas de acción que tales políticas tienen que tomar en cuenta es precisamente la de desarrollar tecnologías educativas apropiadas. La tecnología más adecuada probablemente no existe todavía, pero están los materiales y las experiencias con qué construirla. No parece fuera de lugar plantear una cambio tecnológico radical en la práctica educativa que lleve a los países de la región a saltarse las tecnologías articuladas en torno al libro y al docente de la escuela clásica. Se trataría de utilizar los avances tecnologicos y de gestión que actualmente se están aplicando en diversos ámbitos de la vida económica y social, especialmente en la esfera de la organización del trabajo y de la acumulación y distribución de la información (Delker, 1990).

7 Aun cuando parses como Perú, cuyos ingresos fiscales son muy bajos, deban buscar estrategias propias, tanto tecnológicas como financieras, para desarrollar su sistema de educación.
El objetivo es producir progreso técnico en la educación, maximizando la eficiencia del capital y del trabajo, pero considerando las restricciones presupuestarias existentes. Es la curva E" del gráfico 1. Probablemente se van a encontrar más combinaciones reales de recursos en estas tecnologías nuevas (E') sobre el segmento B' C', que las que se encuentran sobre el segmento $\mathrm{BC}$, o el segmento $\mathrm{B}^{\prime} \mathrm{C}^{\prime}$ en las curvas E y E', que representan las tecnologías más tradicionales.

La ubicación de las tecnologías reales sobre uno u otro de los segmentos de esas curvas está determinada por restricciones técnicas. En todos los avances tecnológicos productivos ocurridos, al menos desde la revolución industrial, se observó una transferencia de los conocimientos a la maquinaria, a los instrumentos de trabajo y a la organización. El sistema de educación cuenta en este momento con los medios para llevar a cabo sustituciones técnicas análogas.

Las innovaciones tecnológicas encaminadas a hacer más eficientes los sistemas educativos requieren que los materiales de enseñanza y las metodologias para su uso sean producidos industrialmente. De esta manera, los medios didácticos se transforman en los depositarios del conocimiento que reciben los estudiantes y éstos pasan a tener un papel más activo.

Las innovaciones tecnológicas como las que implican uso más intensivo del capital (y con ello una mayor rentabilidad de una mano de obra altamente calificada), no siempre obligan a instalar en cada escuela aparatos, instrumentos o infraestructura especialmente onerosos. A veces basta con una reorganización física de la sala de clases y el uso de materiales apropiados. Hay tecnologías "livianas", basadas principalmente en la reorganización de los medios docentes, con una fuerte contribución externa. También hay, o puede llegar a haber, tecnologías "pesadas" que significan incorporar instrumentos y aparatos, especialmente electrónicos y de computación, al proceso de aprendizaje.

La "escuela nueva" de Colombia, por ejemplo, es una estructuración de la escuela primaria sobre la base de principios de organización análogos a los de la "especialización flexible", como es por ejemplo la 
disposición de la sala de clases en unidades asimilables a las células de producción (Kaplinski, 1984).

La "escuela nueva" ha logrado cambiar el papel del maestro. En ella el docente ya no tiene que dedicar todo su tiempo a transmitir instrucciones o conocimientos que pueden estar escritos en un texto, sino que se asegura que cada alumno esté participando activamente en la experiencia de aprendizaje. Para ello, los alumnos disponen de textos de autoinformación, en los cuales hay instrucciones detalladas sobre cada una de las etapas de la actividad que es necesario realizar para que tenga lugar una experiencia de aprendizaje de real valor. Esas instrucciones son preparadas por centros especializados en desarrollo y contenido curriculares, y llegan directamente a cada alumno, el que las estudia en conjunto con un grupo de tres o cuatro compañeros, y recurre al maestro cuando tiene dificultad para entender algo de ellas. Así, los materiales pedagógicos se preparan para un grupo grande de escuelas, incorporando los avances científicos y pedagógicos y aprovechando las economías de escala. Al mismo tiempo, liberan tiempo del docente, al reducir el que éste debía usar para "transmitir" instrucciones e informaciones.

La "escuela nueva" ha mostrado ser pedagógicamente muy eficiente, logrando resultados satisfactorios con un gasto que es solo un $15 \%$ superior al de las escuelas primarias tradicionales (Schiefelbein, 1992). El proyecto MECE en Chile es otro experimento que trata de introducir diversidad al sistema escolar básico. El mérito de experiencias como éstas es su bajo costo adicional, la prescindencia de la tradicion clásica y la independencia del universo cultural europeo, al cual pertenece la escuela clásica.

Innovaciones de otro estilo son las que se articulan en torno a los computadores personales. Estos, la fibra óptica, los satélites de comunicaciones, etc., dan acceso a información anteriormente vedada al estudiante común, y a relaciones interactivas a distancia. Los costos actuales de estas tecnologías son bajos y tienden a disminuir. En la actualidad, un computador de gran potencia cuesta menos de 1000 dolares, se amortiza en cinco años $y$, usado por cinco estudiantes, tiene un costo por estudiante de $\mathbf{4 0}$ dolares por año. Estos costos pueden ser más bajos si se negocia bien con los fabricantes. Lo mismo sucede con los programas y la información. Resulta hoy más barato un computador con CD-Rom y un disco compacto con una enciclopedia, que la misma enciclopedia en libros.

Experiencias como la "escuela nueva", o como la práctica escolar organizada en tomo a los medios electrónicos de información, imponen relaciones entre los estudiantes y de ellos con los docentes completamente distintas a las que se dan en la escuela clásica. Los estudiantes tienen que habituarse, entre otras cosas, a trabajar en grupo, definir problemas, resolverlos y buscar la información pertinente. Los docentes dejan de ser los depositarios centrales del conocimiento y pasan a ser consultores metodológicos y animadores de los grupos de trabajo.

Esta estrategia obliga a reformular los objetivos de la educación. El desarrollo de competencias claves (formulación de problemas, búsqueda de información, capacidad de medir y de clasificar, pensamiento logico, capacidad de aprender, etc.) reemplaza el objetivo anterior de formación disciplinaria sólida. El uso de nuevas tecnologías educativas lleva a desdibujar los límites entre las disciplinas, redefiniendo al mismo tiempo la función, la formación y el perfeccionamiento de los docentes.

Al mismo tiempo, es preciso estructurar la gestión de los sistemas educativos de modo de incorporar otros sectores que se ven afectados por su rendimiento y que pueden hacer contribuciones valiosas. Esta ampliación de la gestión del sistema está estrechamente ligada a tecnologías pedagógicas más participativas, como lo muestra la experiencia de la educación dual en Alemania y de la "escuela nueva" en Colombia. Permite además establecer una relación entre los contenidos temáticos enseñados en la escuela y las características del desarrollo en la región; se trata de "instalar" las disciplinas o áreas temáticas con la contribución del entorno. Por ejemplo, conviene obtener el apoyo de la industria química local para delinear la enseñanza de esta disciplina en las escuelas cercanas, de los museos para enseñar arte, de la comunidad angloparlante residente para el aprendizaje del inglés, etc. Los medios de comunicación pueden desempeñar un papel central en la instalación escolar de ciertas areas del conocimiento.

Cualesquiera sean la o las tecnologías educativas que se adopten o los cambios que se introduzcan, hay que apuntar a saltar etapas y moverse hacia la frontera tecnológica mundial.

Para ello, los países de la región tienen ventajas sobre los europeos. Paradojicamente, porque su desarrollo cultural es menor y porque los productos y las instituciones de la cultura de referencia son menos accesibles que en los países industrializados, los países de la región están mejor preparados para un cambio radical en las instituciones educativas. Un ambiente cultural muy desarrollado tiene ventajas para 
la escuela clásica y para otras que están inspiradas en ella, pero también puede ser un obstáculo a la innovacion.

Como se ha señalado más arriba, el costo mayor de una educación clásica, y una de sus piezas centrales, es el docente. Aqui no hemos tomado en cuenta el costo de su formación. Pero considerando sólo sus salarios en condiciones adecuadas de aplicación de la escuela clásica o de derivaciones de ella, como es la escuela comprensiva, se llega a la conclusión de que estas modalidades están fuera del alcance de los países de la región. Por lo demás, es siempre más difícil au- mentar la productividad de tecnologías que hacen uso intensivo de los recursos humanos, como son las utilizadas por estos sistemas de educacion. El problema se complica aún más si lo que se busca es mejorar la calidad del producto; las mejoras cualitativas intentadas en los países de la $O C D E$ en los últimos veinticinco años sobre la base de bajar el número de alumnos por docente y mejorar la infraestructura y los materiales, han sido muy insatisfactorios (OCDE, 1992). Algunas nuevas formas de organización escolar y los medios electrónicos permitirían bajar los costos y organizar una educacion que responda a las necesidades actuales.

\section{VII}

\section{La innovación tecnológica en la educación}

\section{y algunas cuestiones de política}

A finales de los años ochenta y en los noventa se han iniciado en la region reformas que pretenden modificar sustancialmente los sistemas educativos. Entre otros, Argentina, Chile, Colombia, Jamaica y México han iniciado ambiciosas transformaciones o se han pronunciado sobre la necesidad de hacerlas.

Estas reformas recientes tienden a superar estrategias de cambio como las que se formularon en los años sesenta y setenta, basadas en mejoramientos de alguna modalidad o de algún nivel, y que perseguian sobre todo mejorar la cobertura del sistema en la modalidad o nivel correspondiente, y actualizar contenidos y programas para responder a demandas internas y externas. En la actualidad se hacen planteamientos de orden más general que apuntan a transformaciones radicales; éstas se reflejan en la formulación de politicas que reasignan roles y funciones a los distintos sectores sociales comprometidos.

Las políticas educativas estructuradas en torno a modalidades o sectores de la educación ofrecían ventajas conceptuales y facilitaban el proceso de toma de decisiones. Las propuestas más recientes de alcance global carecen de estas precisiones, y a menudo se diluyen en pronunciamientos generales de confusa o difícil implementacion. La política se queda entonces en lineamientos generales, descuidando asuntos "prácticos" ligados a la implementacion, entre los que destacan los presupuestarios y tecnologicos.

Una estrategia realista que aspire a introducir las modificaciones necesarias e integrar a la vez los principios generales, debe incluir las dimensiones señaladas en el proceso de estructuración del sistema educativo. Esto se traduce en las siguientes políticas y correspondientes líneas de acción:

\section{Política A}

Optar claramente por avanzar hacia la frontera tecnologica, usando los avances de la pedagogía, la electrónica, la computación y la comunicación. Las líneas de acción serían:

i) Optar por tecnologías educativas que muestren una alta relación efectividad/costos y que, al mismo tiempo, sustituyan trabajo por capital, ya sea en el proceso de confección de materiales de apoyo a la docencia o en la organización y gestión de la docencia misma.

ii) Reducir la brecha tecnologica entre los sistemas educativos de la región y los de los países industrializados, por medio del empleo de tecnologías educativas adecuadas a los parses de la región.

iii) Estimular la diversidad al interior del sistema educativo, desarrollando las disciplinas o temas en las zonas geograficas donde eso sea más promisorio, y los métodos pedagógicos más adecuados. 


\section{Politica B}

Incentivar la transferencia, la adaptación y el desarrollo de tecnologías educativas que tengan en cuenta las restricciones presupuestarias existentes. Las líneas de acción serian:

i) Apoyar la formación de alianzas estratégicas entre los sectores comprometidos (docentes, funcionarios del ministerio de educación, asociaciones de alumnos y de padres); incorporar sectores nuevos, como empresas (productoras de material electrónico y comunicaciones) y medios de comunicación (radio, televisión y prensa escrita), y permitir la participación más activa de los sectores académicos.

ii) Buscar la transferencia de la tecnología educativa de vanguardia ya desarrollada en el exterior. Esto significa buscar la información pertinente y crear o estimular organismos con la misión de traducir y adaptar metodologías, procedimientos, textos, etc.

iii) Organizar programas para generalizar la difusion de las mejores tecnologías educativas en la región.

iv) Establecer redes de información interactivas que permitan una comunicación fluida entre todos los interesados en mejorar la calidad de la educación.

v) Seguir de cerca las experiencias innovativas más prometedoras, con evaluaciones periódicas en función de objetivos y estándares de calidad, y difusión de los resultados de tales evaluaciones.

\section{Politica $\mathrm{C}$}

Mejorar el aporte presupuestario estatal a la educación, tanto cualitativa como cuantitativamente. Las líneas de acción serían:

i) Aumentar el presupuesto estatal para la educación, sin buscar sustituirlo con aportes privados.

ii) Distribuir el presupuesto estatal con objetivos posibles de alcanzar, para lo cual es conveniente hacer estudios de costo/beneficio en función de objetivos muy precisos, y definir la estrategia más eficiente. iij) Ofrecer estímulos fiscales a la investigación e innovación, superando la práctica actual de incentivar casi exclusivamente la matrícula.

iv) Delimitar el campo de lo privado y de lo público en materia de educación. La definición de las habilidades básicas que caen bajo la responsabilidad del Estado debe ser un punto de partida para esa definición.

\section{Política D}

Establecer estructuras estatales, regionales, nacionales o locales, capaces de llevar a cabo estas políticas. Esto con el objeto de superar el inmovilismo de la mayoría de los ministerios de educación en la región. Las líneas de acción serían:

i) Crear organismos coordinadores a nivel local, con atribuciones para integrar a todos los sectores interesados y comprometidos con el sector. Estos organismos deben tener además la capacidad de tomar iniciativas en materia de investigación e implementación de innovaciones pedagógicas.

ii) Crear organismos de asesoría permanenente $u$ ocasional a los establecimientos que lo soliciten en materia de innovaciones pedagógicas y de gestión.

iii) Tener una preocupación especial por prevenir trastornos y desajustes mayores causados por el cambio. El estudio de experiencias similares puede ser muy instructivo.

\section{Política E}

La formación tanto de los nuevos docentes como de aquéllos en ejercicio para aplicar las nuevas tecnologías es un punto estratégico central en cualquier política de innovación. Las líneas de acción serían

i) Buscar la integración de institutos y universidades pedagógicos al desarrollo de tecnologías educativas y a la formulación de políticas para el sector.

ii) Visitas y estadas de docentes para conocer las experiencias educativas más avanzadas dentro y fuera del país. 


\section{Bibliografia}

Banco Mundial (1993): Informe sobre el desarrollo mundial 1993, Washington, D.C., Oxford University Press.

CEPAl (Comisión Econónica para América Latina y el Caribe) (1990): Transformacion productiva con equidad, LC/G. 1601-P. Santiago de Chile. Publicación de las Naciones Unidas, $\mathrm{N}^{\circ}$ de venta S.90.II.G.6.

(1993): Anuario Estadistico de América Latina y el Caribe. Edicion I992, LC/G. 1747-P, Santiago de Chile. Publicación de las Naciones Uuidas, $\mathbf{N}^{\circ}$ de venta S.93.II.G.I.

Coleman J. (1966): Equality and Educational Opportunity, Washington D.C., U.S. Government Printing Office , 1966.

Delker, W.A. (1990): Basic Skills Education in Business and Industry: Factors for Success or Failure, Washington D.C. Congreso de los Estados Unidos, Oficina de Evaluación Tecnologica.

Duran-Drouhin, M. (1993): Recent Developments and Policy Debate Concerning Post Compulsory and Upper Secondary Education and Training. trabajo presentado al seminario Educación y Trabajo, Santiago de Chile, Centro de Investigación y Desarrollo de la Educación (CIDE), mimeo.

Ferreyra, M. (1993): La educación tecnológica, tendencias actuales, documento presentado en el seminario Educación y Trabajo, Santiago de Chile, CIDE, mimeo.

Jencks, C. (ed) (1972): Inequality, Nueva York, Basic Books

Johnson, B. y B.A. Lundvall (1988): Institutional Learning and
National Systems on Innovation, Roskilde, Dinamarca, Roskilde Universitetscenter, mimeo.

Johnson, L. y V. Packer (1987): Workforce 2000, Work and Workers for the 21th Century, Indianapolis, The Hudson Institute.

Kaplinsky, R. (1984); Atutomation. The Technology and the Society, Londres, Longman.

MECE 1.2 (Proyecto Mejoramiento de la Calidad y Equidad de la Educación) (1993): La experiencia internacional en el diseño curricular, Santiago de Chile, cIDE/Ministerio de Educación Pública.

OCDE (Organización de Cooperación y Desarrollo Económicos), (1992): Public Educational Expenditure, Costs and Financing, Parts.

Peres, W. (1994): Políticas de Competitividad, Revisfa de la CEPAL, N 33, LC/G. 1832-P, Santiago de Chite.

Schiefelbein, E. (1992): En busca de la escuela del S. XXI, Santiago de Chile, Corporacion de Promoción Universitaria (CPU) Oficina Regional de Educación de la UNESCO para América Latina y el Caribe (OREALC).

UNESCO (Organización de las Naciones Unidas para la Educación, la Ciencia y Ia Cultura) (1992): Amuario Estadistico 1992. París.

(1993): Anuario estadístico 1993, París. (1994): Anuario estadístico 1994, París. 